\title{
SURGICAL OUTCOME OF TIBIALIS POSTERIOR TENDON TRANSFER FOR FOOT DROP IN LEPROSY PATIENTS
}

\author{
Vijay Krishna ${ }^{1}$, Jenson Isaac ${ }^{2}$, Shanmuga Vel3 ${ }^{3}$ Ranjith Kumar 4 , Bindhu Rajkumar ${ }^{5}$ \\ ${ }^{1}$ Senior Resident, Department of Orthopaedics, Tagore Medical College Hospital and Research Institute. \\ ${ }^{2}$ Assistant Professor, Department of Orthopaedics, Tagore Medical College Hospital and Research Institute. \\ ${ }_{3}^{3}$ Senior Resident, Department of Orthopaedics, Tagore Medical College Hospital and Research Institute. \\ ${ }_{4}^{4}$ Senior Resident, Department of Orthopaedics, Tagore Medical College Hospital and Research Institute. \\ ${ }_{5}^{5}$ Senior Resident, Department of Orthopaedics, Tagore Medical College Hospital and Research Institute.
}

\section{ABSTRACT}

Leprosy is the most common treatable cause of neuropathy in the world.[1] The social stigma in leprosy is mainly due to nerve function impairment and subsequent disability. Loss of common peroneal nerve function leading to paralysis of the anterior and lateral compartment muscle groups in the leg is common in leprosy. Surgical correction of foot drop in leprosy is done by anterior transposition of the tibialis posterior tendon. The aim of the study is to assess the functional outcome of tibialis posterior tendon transfer procedure and to identify factors that impact favourable outcome of tibialis posterior tendon transfer.

\section{METHODS}

Our study is a prospective study of 50 leprosy patients who underwent tibialis posterior tendon transfer between Jan 2012 to Dec 2015 in Tagore Medical College Hospital. In our study of 50 patients, 44 were males and 6 were females with sex ratio 22:3. The maximum number of cases who underwent surgery were between 26 to 35 age group; 45 cases were borderline tuberculoid type (90\%) and 5 cases were borderline lepromatous type (10\%); 44 cases underwent interosseous route tendon transfer and 6 cases by circumtibial route tendon transfer.

\section{RESULTS}

By modified daf grading, $80 \%$ of patients in our study had good-to-excellent results; 43 patients (86\%) in our study had normal heel-toe gait after surgery; 15 patients developed inversion deformity of foot and 5 patients developed talonavicular collapse.

\section{CONCLUSION}

The usefulness of tibialis posterior tendon transfer as a method of correction of foot drop in leprosy is well known. In our study the gains of normal gait, favourable foot at rest position and active range of movement in the dorsiflexion range were improved after tendon transfer surgery. In the long-term evaluation of this procedure where the follow-up ranged from 2 to 10 years, tibialis posterior transfer functioned well and did not develop major surgery related complications like neuropathy, loosening of transfer by stretching of tendon or occurrence of new deformities. Apart from improving the gait, tibialis posterior transfer helped prevent recurrent ulceration in anaesthetic feet also.

\section{KEYWORDS}

Tibialis posterior, Foot drop, Leprosy.

HOW TO CITE THIS ARTICLE: Krishna V, Isaac J, Vel S, et al. Surgical outcome of tibialis posterior tendon transfer for foot drop in leprosy patients. J. Evolution Med. Dent. Sci. 2016;5(52):3395-3399, DOI: 10.14260/jemds/2016/784

\section{INTRODUCTION}

Leprosy is the most common treatable cause of neuropathy in the world.[1] In all patients with leprosy, the nerve tissue is involved. The dermal nerves are infected in all skin lesions including those due to indeterminate leprosy of childhood. Clinical examination is often sufficient to reliably diagnose leprous neuropathy.

Leprosy is a common cause of neuropathy in developing countries, although it is also seen in developed countries. The prevalence of leprosy is gradually declining. The registered prevalence as of December 2006 was 220,741 cases, of which 116,663, are from Asia.[2]

Financial or Other, Competing Interest: None.

Submission 29-05-2016, Peer Review 10-06-2016,

Acceptance 13-06-2016, Published 29-06-2016.

Corresponding Author:

Jenson Isaac,

Flat No. 4041,

Estancia Township

Guduvanchery, Chennai-603202.

E-mail: docjenson@gmail.com

DOI: 10.14260/jemds/2016/784
Brazil, Democratic Republic of Congo, Nepal, Mozambique and Philippines are some major countries with a prevalence of greater than 1 case per 10,000 inhabitants.

About 1-2 million people are visibly and irreversibly disabled because of past or present leprosy. Of patients with LL disease, $70-75 \%$ have an eye, hand and/or foot disabilities. ${ }^{[3]}$ According to one study, the frequency of nerve function impairment at the presentation in regions of endemic disease is 1.7 per 100 patient-years in PB leprosy and 12 per 100 patient-years in MB leprosy.[4]

Loss of common peroneal nerve function leading to paralysis of the anterior and lateral compartment muscle groups in the leg is common in leprosy. Leprosy neuritis affects nerve where they are close to skin and pass through a narrow fibro-osseous canal. In the leg this involves the lateral popliteal nerve at the neck of the fibula, which leads to foot drop and the posterior tibial nerve in the tarsal tunnel which produces anaesthesia of the sole. When both nerves are damaged, the main impact of walking falls on anaesthetic forefoot rather than on the heel and causes trophic ulceration. 
In addition to common peroneal nerve paralysis, there is often associated loss of posterior tibial nerve function leading to loss of the intrinsic muscles of the foot. This paralysis leads to hyperflexion at the distal and proximal interphalangeal joints (Claw toes), which may remain after tibialis posterior transfer. In some cases, a transfer of flexor to extensor tendon in the toe may be required or if there is fixed non-correctable damage to the toe fusion is indicated.

The aim of the study is to assess the functional outcome of tibialis posterior tendon transfer procedure and to identify factors that impact favourable outcome of tibialis posterior tendon transfer.

\section{MATERIALS AND METHODS}

This is a prospective study of leprosy patients who underwent tibialis posterior tendon transfer between Jan 2012 to Dec 2015 at Tagore Medical College Hospital and Research Institute. The objective of the study is to assess the functional outcome of tibialis posterior tendon transfer and to identify factors that impact favourable outcome of tibialis posterior tendon transfer. After getting clearance from Academic Council and Ethical Committee Clearance, information required for the study were obtained from clinical record and subsequent follow-up. At the time of admission, a detailed history of the patient was recorded with regards to the duration of the disease, duration of paralysis. Clinical examination was done to classify the disease, presence or absence of leprosy reaction, degree of paralysis, sensory assessment and presence of secondary changes in the paralysed foot.

The range of motion at the ankle joints is recorded using goniometer. The foot at rest position and the tendon Achilles contracture are assessed and recorded. The patients are then taught to isolate action of the tibialis posterior tendon to be transferred until it stands out on command. The preoperative physiotherapy usually takes about 7 days.

\section{Surgical Technique}

Both Interosseous \& Circumtibial routes have been surgical choice for tibialis posterior tendon transfer. The most preferred method of choice is Interosseous route. Achilles tendon lengthening procedure is first performed by the subcutaneous Z-lengthening. An $8 \mathrm{~cm}$ curved or straight incision is made starting one to two finger breadths proximal to the medial malleolus in the line of the tibialis posterior tendon and the tibialis posterior tendon exposed and isolated. The tibialis posterior tendon is identified in the lower half of the leg lying deep to flexor digitorum longus tendon. Care is taken not to damage the long saphenous vein or nerve.

The tendon is isolated in the leg and placed on a flat scissor and confirmed by pulling on it above the malleolus. The tendon is then divided at its insertion, freeing it close to its attachments to maintain length. Tibialis posterior tendon is delivered into leg from the foot. Using a tunneller the tendon is routed through a window made through another $7 \mathrm{~cm}$ incision in the anterior compartment in the leg. The tendon is then divided into two equal slips up to the musculotendinous junction. Each slip is then tunnelled to EHL and EDL under the extensor retinaculum using a tunneller distal to the axis of the ankle joint. With the knee flexed the ankle is held in at least 20 degrees dorsiflexion (By assistant or using Fritschi splint) each slip is anastomosed to EHL and EDL.
The anastomosis is done by the double weave technique. The tension between the two slips should be equal and foot should be inadequate dorsiflexion. The wounds are dressed and a below knee cast applied with the foot in neutral and 15 to 20 degrees of dorsiflexion for 3-4 weeks, after which physiotherapy commenced.

\section{Post-Op Physiotherapy}

The plaster cast was left intact for 5 weeks. Since the introduction of the tendon-to-tendon insertion methods, it is not considered necessary to immobilise the foot for more than 5 weeks. At the end of 5 weeks, the plaster cast is removed and the posterior half of the bivalved cast is reapplied as a nonweight bearing splint, which is worn constantly outside training periods for the next 10 to 14 days. The sutures are removed, and unless some failure in union is evident active physiotherapy is instituted. Three to four weeks are the usual period of postoperative physiotherapy, after which the patient is discharged with instructions to return later for follow-up studies and final evaluation. As the patient gains confidence in the daily use of the transferred muscle, the careful, deliberate overuse is changed into a more normal gait.

\section{OBSERVATION AND RESULTS}

Our study is a prospective study of 50 leprosy patients who underwent tibialis posterior tendon transfer between Jan 2012 to Dec 2015 in Tagore Medical College Hospital. In our study of 50 patients, 44 were males and 6 were females with sex ratio 22:3. Maximum number of cases who underwent surgery were between 26 to 35 age group; 45 cases were borderline tuberculoid type (90\%) and 5 cases were borderline lepromatous type (10\%) (Figure 1).

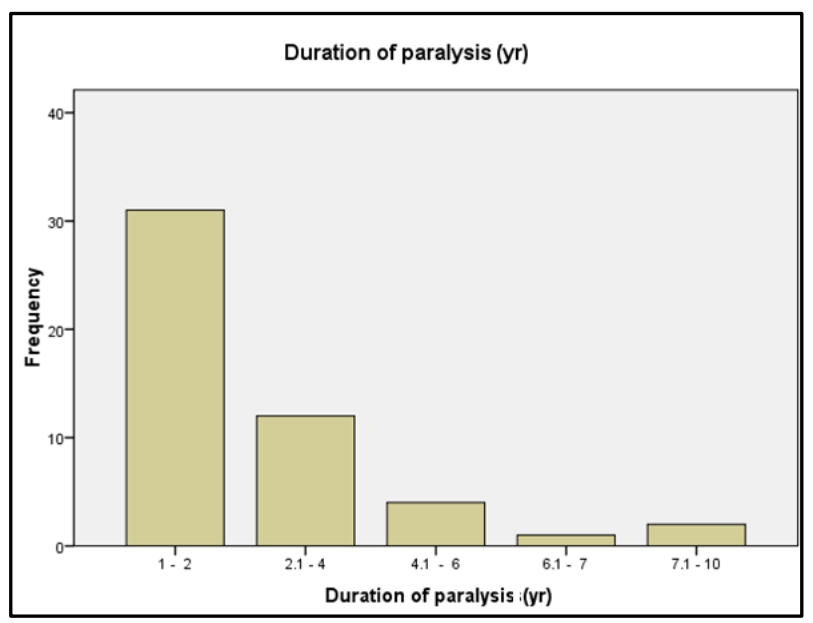

Fig. 1: Duration of Paralysis in our Patients

44 cases underwent interosseous route tendon transfer and 6 cases by circumtibial route tendon transfer. Double slip tendon transfer with end-to-end anastomosis was done to 48 patients and single slip tendon transfer done for 2 patients (Table 1).

\begin{tabular}{|c|c|c|c|c|}
\hline & $\begin{array}{c}\text { Frequ- } \\
\text { ency }\end{array}$ & Percent & $\begin{array}{c}\text { Valid } \\
\text { Percent }\end{array}$ & $\begin{array}{c}\text { Cumulative } \\
\text { Percent }\end{array}$ \\
\hline CT DOUBL & 6 & 12.0 & 12.0 & 12.0 \\
\hline IO DOUBL & 42 & 84.0 & 84.0 & 96.0 \\
\hline IO SINGL & 2 & 4.0 & 4.0 & 100.0 \\
\hline Total & $\mathbf{5 0}$ & $\mathbf{1 0 0 . 0}$ & $\mathbf{1 0 0 . 0}$ & \\
\hline \multicolumn{5}{|c|}{ Table 1: Single or Double Slip } \\
\hline
\end{tabular}


By modified daf grading $80 \%$ of patients in our study had good-to-excellent results, 43 patients (86\%) in our study had normal heel-toe gait after surgery; 15 patients developed inversion deformity of foot and 5 patients developed talonavicular collapse.

Range of active dorsiflexion is calculated by the following criterion: criterion for grading (Table 2 and 3 ).

\begin{tabular}{|c|c|}
\hline$>15$ & Grade 1 \\
\hline 10 to 15 & Grade 2 \\
\hline 5 to 9 & Grade 3 \\
\hline 0 to 4 & Grade 4 \\
\hline \multicolumn{2}{|c|}{ Table 2: Criterion for Grading } \\
\hline
\end{tabular}

\begin{tabular}{|c|c|c|c|c|}
\hline & $\begin{array}{c}\text { No. of } \\
\text { Feet }\end{array}$ & Percent & $\begin{array}{c}\text { Valid } \\
\text { Percent }\end{array}$ & $\begin{array}{c}\text { Cumulative } \\
\text { Percent }\end{array}$ \\
\hline Grade 1 & 5 & 10.0 & 10.0 & 10.0 \\
\hline Grade 2 & 35 & 70.0 & 70.0 & 80.0 \\
\hline Grade 3 & 9 & 18.0 & 18.0 & 98.0 \\
\hline Grade 4 & 1 & 2.0 & 2.0 & 100.0 \\
\hline Total & $\mathbf{5 0}$ & $\mathbf{1 0 0 . 0}$ & $\mathbf{1 0 0 . 0}$ & \\
\hline \multicolumn{6}{|c|}{ Table 2\&3: New dafGrade } \\
\hline
\end{tabular}

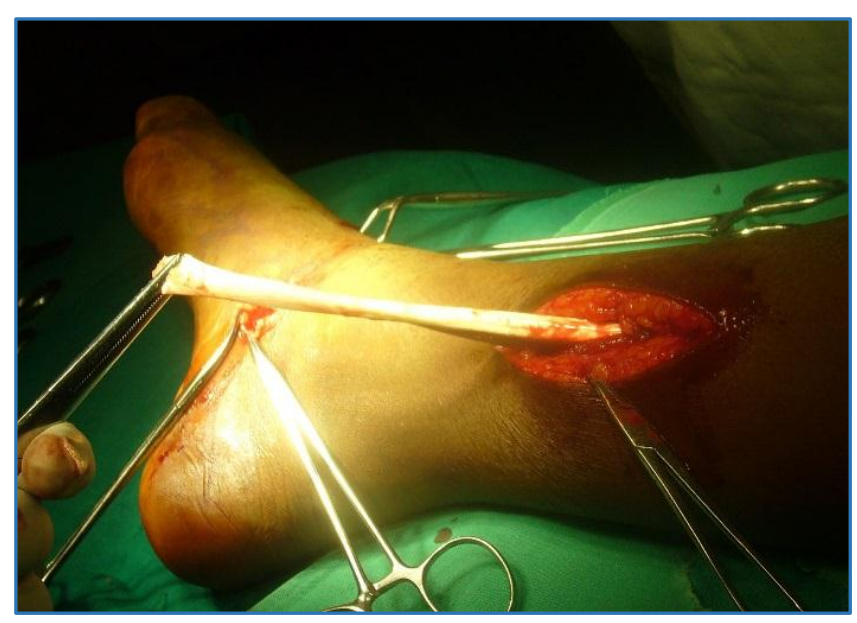

Fig. 2: Tibialis Posterior Tendon is Delivered into Leg from the Foot

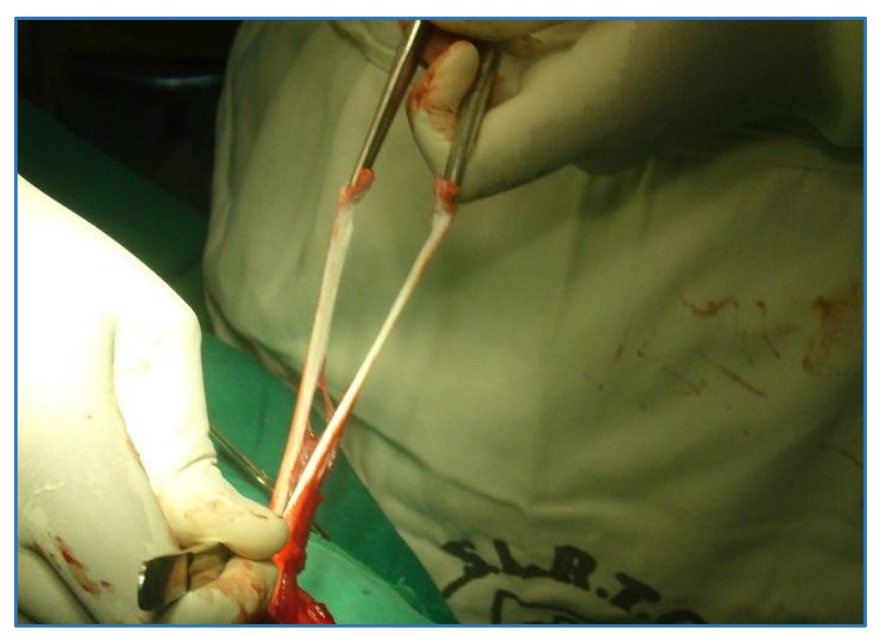

Fig. 3: The Tendon is Divided into Two Equal Slips upto Musculotendinous Junction

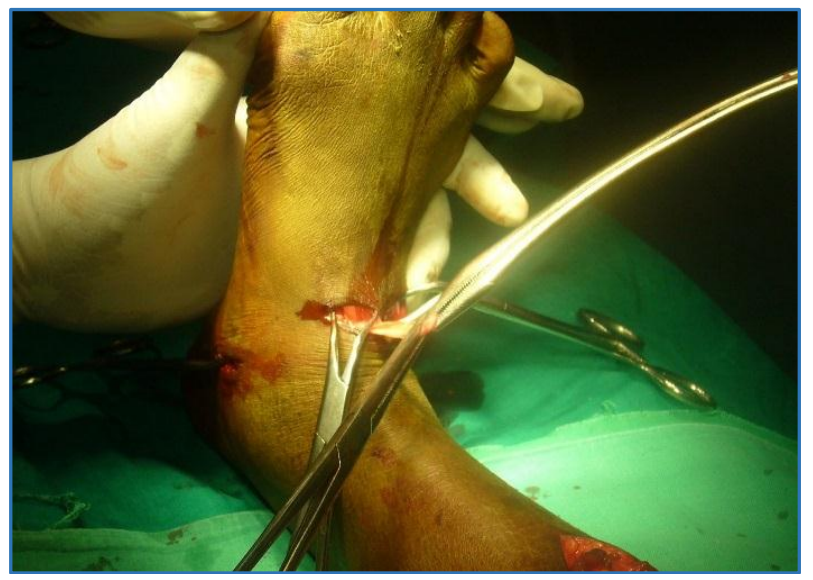

Fig. 4: The Anastomosis is Done by a Double Weave Technique

\section{DISCUSSION}

Leprosy is a chronic disease with many social implications. Social stigma in leprosy is mainly due to nerve function impairment and subsequent disability. Surgical correction of foot drop in leprosy appears to have been neglected until Brand (1955) pointed out the anterior transposition of the tibialis posterior tendon to the intermediate cuneiform bone gave encouraging results. Since then there have been a number of reports of the use of this operation with or without modification.

Gunn and Molesworth (1957) reported fifty six cases of drop-foot, in which the tibialis posterior tendon was inserted into the tarsus after being brought forward the interosseous membrane of the leg.

Carayon.[5] Bourrel, Bourges and Tourz'e (1967) reported twenty-three cases of drop foot (18 caused by leprosy), in which they had done dual transfer of the tendons of the tibialis posterior and flexor digitorum longus.

The procedure used in our study was interosseous route, subcutaneous, two-tailed, tendon-tendon transfer of the tibialis posterior-to-extensor hallucis longus and to extensor digitorum longus and peroneus tertius. The motor slips were inserted into the recipient tendons on the dorsum of the foot. The outcome measures depend on several factors and the influence of these factors on the results is discussed.

The sex ratio was 22:3 with male (44) and female (6) and the percentage value of male (88\%) and female (12\%). Increased outdoor activities and subsequent exposure in males and lower case detection rate due to incomplete screening of females. Maximum number of cases undergone between age group 26-35 yrs. is 18 cases with $18 \%$. Age and Gender had no significant effect on outcome. Even the type of disease may not significantly affect the outcome independently in our study.

The maximum no. of cases undergone surgery is 2 yrs. duration of paralysis and minimum no. of cases undergone is between 6 to 10 yrs. duration of paralysis respectively. Stable paralysis of not less than 6 months duration has no chances of recovery by Johs G Anderson et al.[6] Recovery of neural damage resulting in foot drop to steroid therapy is documented well. It is now known that lateral popliteal nerve paralysis of less than 9 months duration should be given a chance to recover with rest and steroids, consecutively no patient should be operated whose paralysis is less than 9 
months duration. Duration of paralysis also had significant effect on patient satisfaction.[6]

The commonest type of surgery done in our study group is the interosseous route method and least common is the circumtibial route method. The interosseous route remains popular for tibialis posterior tendon transfer). Only two series give objective clinical data: Hall (1977) and Uchiyama (1981) described postoperative angles of active dorsiflexion and range of movement and correlated them with functional results.[7] Tibialis posterior transfer for the treatment of drop foot was advised probably for the first time by Ober in 1933. The several modifications of the technique of transfer suggests that it does not always work. This possibly is because of the difficulty in training the transferred muscle to act in a movement phase when it normally is relaxed. From our study, we find that the range of movement gets stabilised by the end of the first postoperative year. However, some cases showing good results initially seem to lose the ability to use the transfer later on. However, after the third postoperative year, it is unusual to come across a failure in a previous good result case. The above indicates the long time required for phase transfer to be completely established.

Analysis of the results showed some correlation between the angle of active dorsiflexion seemed to determine the range of movement. Tibialis posterior transfer done by either route plus elongation of tendo Achilles will produce active dorsiflexion in $80 \%$ of patients with the restoration of nearnormal gait in $94 \%$. The Circumtibial route, however, was associated with an unacceptably high rate of recurrent inversion leading to ulceration of the lateral border of the foot. This observation was also made in small subgroups of patients by Richard BM.[8] Circumtibial transfer should be reserved for patients with a calcified and unyielding interosseous membrane; these are usually elderly with recurrent inflammation and infection in the foot. If this type of transfer is performed, the tendon bifurcation must be at least $3 \mathrm{~cm}$ above the ankle so that the line of pull is as close to the vertical as possible. Where possible an interosseous route should be used. There was no evidence in this series of the 'crippling adhesions' said to be associated with the IO route.[9] and at final follow-up the interosseous route produced better active dorsiflexion, although less active plantar flexion than circumtibial route.

The insertion of tendon slip can be single or double slip depending on the assessment of patient and surgeon's choice. The commonest done method of choice is interosseous double slip technique, other methods also followed such as interosseous single slip technique, circumtibial double slip technique. Many surgeons continue to fix the transfer into an osseous tunnel in the lateral cuneiform. This has two disadvantages: first, it has been shown to increase the likelihood and severity of the tarsal joints in predisposed patients. ${ }^{[9]}$ Secondly, the fixation point is critical in ensuring that there is no tendency for later inversion or eversion deformity. The tendon-to-tendon technique of Srinivasan.[10] et al (1968) obviates these problems, allowing the surgeon to adjust the tension on the two tails appropriately. The benefits from the different techniques are only marginal when as dorsiflexion is concerned. The papers cited also show that tendon-to-tendon fixation is better and that it is not imperative to do tendon-to-bone fixation. The need therefore is the least complicated procedure, which will work well.
In our study, 7 patients had high stepping gait and the rest 43 patients had normal heel-toe gait.

While evaluating our failure cases, it became apparent that in spite of having good range of dorsiflexion as an isolated movement, these cases failed to achieve good heel-toe gait. It seems that there are two types of post-operative failures - one the failure of technique and another the failure to achieve central integration of the movements of ankle as a whole because of lack of constant feedback and education, thus depriving the patient of a good heel-toe gait. Much of the success of the procedure depends on how we teach the correct use of transfer to the patient. Physiotherapy thus plays a very important role in the final outcome of surgery.

H. Srinivasan et al[10] S. M. Mukherjee et al and R. A. Subramaniam et al showed the criteria used were angle of active dorsiflexion at the ankle, range of active movement of the foot. The ankle was also graded independently according to their range of active movement. This being the difference between the angles of active plantar flexion and of active dorsiflexion. Ankles with a range active movement of 10 degrees or less were graded as 3 ; those with a range of 15 or 20 were graded as 2 ; those with a range of 25 or more were graded as grade 1 .

The range of active dorsiflexion after Tibialis Posterior Tendon Transfer in 50 patients had the following results. Among 50 Feet.

- 35 had grade 2 value with $70 \%$.

- 9 Feet had grade 3 value with $18 \%$.

- 5 Feet had grade 1 value with $10 \%$.

- 1 Feet had grade 2 value with $2 \%$.

\section{COMPLICATIONS}

10 patients developed inversion deformity of foot out of fifty patients. The commonest complications encountered are as follows: 1) Inadequate elevation of the foot ( 2 cases), because of undue lengthening of the tendon; 2) Inversion or eversion of the foot (10 cases) related to the placement of the new insertion and the pull on any remaining muscles; 3) Talonavicular collapse ( 2 cases). In reconstructive surgery the first indication occurs some two to four weeks after the removal of the plaster, when the patient starts to walk again. The foot becomes warm and slightly swollen, but there is usually no discomfort.[11] Job 1964, Harris.[12]; 4) Dropped toes caused by laxity of the tendons of the paralysed extensor digitorum longus ( 1 case) when the ankle once again assumes the right angled position and pull of the tendons of the flexor digitorum longus shortened by the dropped foot position. This may cause the patient to walk on the nails of the toes, which are turned under; 5 ) Clawed toes (1 case) which may result if too strong a pull is to the toe extensors by suturing these to the mobile section of the active tendon-muscular transfer when there are no muscles to provide flexion of the metatarsophalangeal joints.[9] (Table 4).

\begin{tabular}{|c|c|c|}
\hline & Frequency & Percent \\
\hline Inversion & 10 & 20 \\
\hline Talonavicular collapse & 2 & 4 \\
Dropped Toes & 1 & 2 \\
Clawed Toes & 1 & 2 \\
\hline Total Table 4: Complications \\
\hline \multicolumn{2}{|c|}{} \\
\hline
\end{tabular}


The foot at rest position and the range of movement, which were seen after surgery were improved.

Postoperative complications were minimal. The interosseous method was better than circumtibial. Tibialis posterior tendon transfer has served the gait and foot function in the long term.

\section{CONCLUSION}

The usefulness of tibialis posterior tendon transfer as a method of correction of foot drop in leprosy is well known. Removal of tibialis posterior tendon, a deforming force (Invertor) in the presence of paralysis of common peroneal nerve, does not cause any deficit. In our study, the gains of normal gait, favourable foot at rest position and active range of movement in the dorsiflexion range seen initially are improved after tendon transfer surgery.

The interosseous group fared better because of a favourable foot at rest position and good dorsiflexion range of movement in the most of its patients.

In the long term evaluation of this procedure where the follow-up ranged from 2 to 5 years, tibialis posterior transfer functioned well and did not develop major surgery related complications like neuropathy, loosening of transfer by stretching of tendon or occurrence of new deformities. Apart from improving the gait, tibialis posterior transfer helped prevent recurrent ulceration in anaesthetic feet.

\section{REFERENCES}

1. Birch R. Surgical disorders of the peripheral nerves. Springer London 2011.

2. Garland DE, Hughston JC. Peroneal nerve paralysis: a complication of extensor reconstruction of the knee. Clin Orthop Relat Res 1979;140:169-71.
3. Goh JC, Lee PY, Lee EH, et al. Biomechanical study on tibialis posterior tendon transfers. Clin Orthop Relat Res 1995;319:297-302.

4. Wolfe SW, Hotchkiss RN, Pederson WC, et al. Green's Operative Hand Surgery E-Book: Expert Consult: Online and Print. Elsevier Health Sciences, 2010:p 2392.

5. Carayon A, Bourrel P, Bourges M, et al. Dual transfer of the posterior tibial and flexor digitorum longus tendons for drop foot. Report of thirty-one cases. J Bone Joint Surg Am 1967;49(1):144-8.

6. Jahss MH. Foot and ankle pain resulting from rheumatic conditions. Curr Opin Rheumatol 1992;4(2):233-40.

7. Uchiyama E, Kitaoka HB, Fujii T, et al. Gliding resistance of the posterior tibial tendon. Foot Ankle Int 2006;27(9):723-7.

8. Richard BM. Interosseous transfer of tibialis posterior for common peroneal nerve palsy. J Bone Joint Surg $\mathrm{Br}$ 1989;71(5):834-7.

9. Andersen JG. Indications and contra-indications in reconstructive surgery in leprosy. Lepr Rev 1963;34: 127-31.

10. Srinivasan H, Mukherjee SM, Subramaniam RA. Twotailed transfer of tibialis posterior for correction of dropfoot in leprosy. J Bone Joint Surg Br 1968;50(3):623-8.

11. Petersen W, Hohmann G, Stein V, et al. The blood supply of the posterior tibial tendon. J Bone Joint Surg $\mathrm{Br}$ 2002;84(1):141-4.

12. Ness ME, Long J, Marks R, et al. Foot and ankle kinematics in patients with posterior tibial tendon dysfunction. Gait Posture 2008;27(2):331-9. 